\title{
Current facts constituting an understanding of the nature of adenomyosis
}

\author{
Veronika Aleksandrovych ${ }^{1, A-F}$, Paweł Basta ${ }^{2, B, C, E, F}$, Krzysztof Gil ${ }^{1, A, D-F}$ \\ ${ }^{1}$ Department of Pathophysiology, Jagiellonian University Medical College, Kraków, Poland \\ 2 Department of Gynecology and Oncology, Jagiellonian University Medical College, Kraków, Poland \\ A - research concept and design; $\mathrm{B}$ - collection and/or assembly of data; $\mathrm{C}$ - data analysis and interpretation; \\ $D$ - writing the article; $E$ - critical revision of the article; $F$ - final approval of the article
}

\section{Address for correspondence}

Veronika Aleksandrovych

E-mail:v.aleksandrovych@doctoral.uj.edu.pl

Funding sources

None declared

Conflict of interest

None declared

Received on September 4, 2017

Reviewed on October 16, 2017

Accepted on 0ctober 25, 2017

Published online on August 7, 2018

\begin{abstract}
Adenomyosis seems to be the most widespread coexistent pathology included under the umbrella of common benign disorders of the human uterus. The incidence of adenomyosis is under discussion since different imaging criteria are used. In the majority of cases, prevalence is determined among women with uterine fibroids and endometriosis or severe gynecological symptoms. This common benign pathology is asymptomatic in $1 / 3$ of cases. Up to $50 \%$ of women with infertility are affected by adenomyosis. It seems to be an important risk factor for spontaneous pre-term delivery and pre-term premature rupture of the membranes. Nowadays, the etiology of adenomyosis is still unclear and requires deeper investigation. This review summarizes the aspects of prevalence, co-existence, risk factors, classification, mechanisms of pathogenesis, genes and immunological features, main histological features, animal models, and clinical manifestation of adenomyosis. It might facilitate understanding of the independent nature of such a dual enigma as adenomyosis.
\end{abstract}

Key words: infertility, adenomyosis, myometrium, metaplasia, endometrial junctional zone

Cite as

Aleksandrovych V, Basta P, Gil K. Current facts constituting an understanding of the nature of adenomyosis. Adv Clin Exp Med. 2019;28(6):839-846. doi:10.17219/acem/79176

DOI

10.17219/acem/79176

\section{Copyright}

Copyright by Author(s)

This is an article distributed under the terms of the

Creative Commons Attribution Non-Commercial License

(http://creativecommons.org/licenses/by-nc-nd/4.0/) 


\section{Introduction}

Adenomyosis is a benign condition of the uterus characterized by the presence of ectopic endometrial glands and stroma below the endometrial-myometrial junction (at a depth of at least $2.5 \mathrm{~mm}$ below the basal layer of the endometrium). ${ }^{1,2}$ The focuses of endometrial glands and stroma in the myometrium are typically surrounded by its hyperplastic tissue. ${ }^{3}$ Lymphatic and vascular channels carry out penetration of normal myometrium. ${ }^{4,5}$ The level of endometrial invasion into a myometrium has been the issue of heated debate. ${ }^{6}$ The majority of cases are observed in multiparous premenopausal women. Likelihood estimation of diagnosis demands obligatory histological analyses, which are commonly provided after hysterectomy. By far, the majority of adenomyosis cases are detected in autopsy and coexist with endometriosis and uterine fibroids, which explain a wide range of distribution (from $5 \%$ to $70 \%$ ) according to literature data. ${ }^{4,7}$

\section{Historical notes}

A Bohemian physician and pathologist, Carl von Rokitansky, first described adenomyoma as a focal core of endometrial glands and stroma surrounded by smooth muscle cells in 1860. He referred to it as "cystosarcoma adenoids uterinum" with a neoplastic nature. ${ }^{8-12}$ Surprisingly, Knapp, an expert in the history of medicine, in 1999 claimed that the $1^{\text {st }}$ who described a similar condition (later named adeno- or endometriosis) was Daniel Schrön from Germany in 1690 in the work "Disputatio Inauguralis Medica de Ulceribus Uteri”. Moreover, some of pathological descriptions made between the $17^{\text {th }}$ and $18^{\text {th }}$ centuries are similar to the modern classification of endometriosis or adenomyosis. Knapp challenged the primacy in describing the pathology in his Letter to Editor of Fertility and Sterility, which was published. Unfortunately, his request remained unanswered due to the author's sudden death within a few months after publication. ${ }^{12}$

The name adenomyoma was coined around the end of the $19^{\text {th }}$ century. In 1896, both Cullen and Von Recklinghausen described the condition, followed by Pick and Rolly in $1897 .{ }^{11}$ In addition, Cullen provided the $1^{\text {st }}$ ever classification of adenomyomas, while its pathogenetic nature was depicted by Meyer in 1903. He emphasized that it is a variant of "epithelial invasion of inflammatory infiltrated tissue" and affects 2 kinds of epithelium: dystopic and orthotopic (embryonic and mature). The origin of dystopic nature was also supported by Orloff, who described it in 1895 as "glandular spaces under the serosa covering uterine myomata", and focused on developing from "embryonic cells". ${ }^{10-12}$

Until the 1920s, adenomyosis and endometriosis were considered to be a part of the same entity. ${ }^{13}$ In 1925, Frankl depicted and named the invasion of mucosal tissue in the myometrium as "adenomyosis uteri", thereby singling out adenomyosis among adenomyomas. ${ }^{10-12}$

Finally, in 1972, Bird proposed the modern explanation of adenomyosis. He described that adenomyosis is a nonmalignant endometrial invasion into the myometrium and leads to macroscopic changes of uterus size. Under a microscope, hypertrophy and hyperplasia of the myometrium are observed with penetration of the endometrium inside (glands and stroma). Nowadays, this gynecological disease is characterized by the invasion of endometrial components more than $2.5 \mathrm{~mm}$ below the endo-myometrial junction in the microscope's low-power field. ${ }^{2,9,12}$

\section{Prevalence and co-existence of adenomyosis}

The incidence of adenomyosis is unclear and debatable due to the use of a variety of criteria. ${ }^{12}$ Numerous publications have shown that the prevalence of adenomyosis widely varies (from $5-8 \%$ to $40-70 \%$ of all uterine specimens), with a mean of $20-25 \% .4,7,8$ The same statistical data was present after histological analysis of postoperative material. Based on a literature review, prevalence of adenomyosis ranges from a high of $61.5 \%$ to a low of $8.8 \%$, with a peak among women between 35 and 50 years of age. ${ }^{7,14}$

The diagnosis of adenomyosis can be time-consuming and challenging, based on lucky histological observation of the material. Interestingly, the rate of detection depends on the quantity and quality of samples observed and can vary from $31 \%$ to $62 \%$ in the same uterus. ${ }^{9}$

Current studies demonstrate that about 4/5 of cases with adenomyosis coexist with uterine fibroids and endometriosis. ${ }^{5,15}$ According to statistical data, approx. every $5^{\text {th }}$ removed uterus with leiomyomata has a focus of adenomyosis, while up to $55 \%$ of adenomyosis samples reveal developing uterine fibroids. Kitawaki proved these conclusions with his own analysis of data presented of the coexistence of both pathologies: adenomyosis with leiomyomata in $35-55 \%$ of cases, while leiomyomata with adenomyosis in $64 \%$. At the same time, Kitawaki noted that adenomyosis is associated with endometriosis too: adenomyosis with endometriosis in $70 \%$ of cases, while "vice versa" only in $6-20 \%$ of all cases. ${ }^{7}$

It is important to note that diseases such as polyps of the endometrium, typical and atypical endometrial hyperplasia and endometrial carcinoma often coexist with adenomyosis, compared to their separate detection. ${ }^{7}$ On the other hand, no study has demonstrated the natural transformation of adenomyosis to adenocarcinoma. ${ }^{16}$

\section{Risk factors}

It is important to consider that adenomyosis often coexists with other gynecological disorders and is revealed by histological examination. As it appears from these 
features, suggested risk factors could not be clearly evaluated and most of them were received after retrospective studies.

Adenomyosis is common for women in the $5^{\text {th }}$ decade of life but it has also been revealed in patients in the postmenopausal period after chemotherapy of breast cancer with tamoxifen. ${ }^{9,17}$ Increasing age up to menopause has long been considered a risk factor. ${ }^{18}$

Multiparity is associated with adenomyosis. This might be explained by a mechanism of trophoblast invasion into the myometrium during pregnancy. ${ }^{9,19}$ Romanek et al. proved by analysis that the relative risk of adenomyosis was almost 2 times lower among nulliparous women in comparison with multiparous. ${ }^{19,20}$ Different studies have presented controversial data regarding abdominal delivery and further risk of pathology development; the role of cesarean sections also remains unclear. ${ }^{19}$

Undoubtedly, sex hormones have a great impact on adenomyosis. ${ }^{21}$ It has been demonstrated that estrogen receptors are always present in adenomyosis tissue, however, their quantity was reduced compared to a corresponding normal myometrium. ${ }^{7}$ Progesterone and androgen receptors were also found in adenomyotic tissue. Some authors have noted a balance between the amount of both steroids in healthy and adenomyotic tissue, while others have demonstrated a slightly higher density of progesterone receptors when compared to estrogen. It is important to note that ectopic as well as eutopic endometrium reflect all cyclic changes. ${ }^{22}$

Several studies have revealed that smoking can also be a risk factor of adenomyosis. Shrestha showed that the risk of adenomyosis is higher in smokers, especially in those who smoked for more than 10 years. ${ }^{20}$ On the other hand, Taran et al. noted that smokers appear less likely to have adenomyosis and this might be explained by declining estrogen levels in the blood, which is common for smokers. ${ }^{7,9}$

Panganamamula et al. focused attention on previous surgery on endometrium, proved the first hypothesis that endometrial resection's involvement in pathogenesis of adenomysosis, made by Coltate and Smith in 1991. ${ }^{23}$ The data rendering a strict direct link between adenomyosis and uterine trauma is still inconsistent. As often as not, in the literature, such traumatic conditions as spontaneous abortion, dilation, curettage, and endometrial ablation are discussed. ${ }^{23}$ Levgur et al. noticed that the instrumental curettage of the uterus could be a risk factor only in the case of abortion, whereas in the nonpregnant uterus it did not impact subsequent development of adenomyosis. ${ }^{4,23,24}$ If it is not expelled, a pregnancy could be a crucial point in the expansion of adenomyosis. One argument in favor of this is the fact that, despite about $20 \%$ of nulliparous women presenting with adenomyosis, a higher percentage of women after any term of pregnancy are affected by the disease. ${ }^{7}$

Some studies have shown that production of prolactin by the endometrium, myometrium and even in leiomyomas could stimulate a mitogenic activity in smooth muscle cells through its receptors. This may suggest a link between adenomyosis and depression with a common pathogenic factor. ${ }^{9}$

\section{Classification}

Adenomyosis has 2 types: diffuse and focal, which is also named "adenomyoma" in the literature. In comparison with the anterior and lateral sides of the uterus, the posterior wall is affected more often. ${ }^{6,12}$

Bird et al. in 1972 divided the depth of invasion into 3 grades. The $1^{\text {st }}$ grade is characterized by the existence of adenomyosis within 1 low-power field below the basal endometrium. The $2^{\text {nd }}$ grade is represented by deeper penetration, to the mid-myometrium, while the $3^{\text {rd }}$ grade penetrates beyond the mid-myometrium. His team assessed the extent (density) of glandular involvement of the myometrium as follows: "slight" - a few (1-3) adenomyotic glands per low-power field; "moderate" - several (4-9) and "marked" - many (10 or more) ${ }^{6}$

\section{Definition of endomyometrial junctional zone}

The endomyometrial junctional zone (JZ) is a distinct, hormonally sensitive part of the endomyometrial interface that was first visualized more than 20 years ago by magnetic resonance imaging (MRI). ${ }^{3,10}$ However, the realization that the inner portion of the human myometrium constitutes a separate entity within the uterine musculature is more than a century old. Brosens et al. referred to Werth and Grusdew, who called it the "archimyometrium" in $1898 .^{25}$

The JZ is not the outer myometrium. ${ }^{12}$ They have different origins: the JZ originates from the Müllerian ductus and the outer myometrium from the mesenchyme. As endometrium, the JZ expresses steroid hormone receptors, has a cycle-dependent type of growth and is involved in implantation and deep placentation. Also, normal uterine peristaltic activity originates from the JZ. This zone has been cited in literature as the archimyometrium, stratum basale, inner myometrium, junctional zone myometrium, endometrio-myometrial interface, transitional zone, and sub-endometrial myometrium. Ultrasound and MRI detect this zone in human uteri. Commonly, the thickness of the JZ is $5 \mathrm{~mm}$ or less, whereas it has a tendency to grow with age. Physiologically, JZ could be up to $0.8 \mathrm{~cm}$ among women without adenomyosis and $1.1 \mathrm{~cm}$ among those affected, respectively. ${ }^{26,27}$

The changing of JZ size and characteristics $(>12 \mathrm{~mm}$, hemorrhagic high-signal myometrial spots) is a poor prognostic for adenomyosis and has a strong correlation with prognosis. ${ }^{25,28}$ It has been called the gold standard in revealing adenomyosis. Bergeron et al. hypothesized that a disturbance of JZ may be involved by triggering an "invasive" factor. ${ }^{5}$ 


\section{Pathogenesis}

The etiology of adenomyosis is still uncertain. Some theories equally explain the possible pathogenesis of the disorder while at the same time complementing each other. They focus on local invasion, cellular proliferation and angiogenesis.

This pathological condition originates from the deep basal layer of the endometrium as a result of its invagination in the myometrium, possibly due to loss of tissue cohesion. Tissue affected by adenomyosis is characterized by higher expression of estradiol receptors (ER) compared to typical endometrium, combined with expression of the apoptosis-suppressing gene product - B cell lymphoma/leukemia-2 (Bcl-2) protein. Enhancement of Bcl-2 expression is involved in the pathogenesis of uterine adenomyosis. ${ }^{5,29-31}$

From another point of view, the basalis invaginates into the myometrium along the lymphatic vessels. This was proved by pathological examination of postsurgical samples - adenomyotic focuses were found within intra-myometrial lymphatics. As the endometrial and myometrial tissues originate from the Müllerian ducts, adenomyosis can be the result of metaplasia from de novo ectopic intramyometrial endometrial tissue. This was defined as the $3^{\text {rd }}$ theory of the pathogenesis of adenomyosis. ${ }^{5}$

It should be noted that myofibroblasts express some extracellular matrix proteins and definitely take part in the development of pathological focuses. The invasion of endometrial tissue inside the myometrium leads to its hypertrophy as a consequent response. ${ }^{28}$ Mast cells in the endometrial tissue are located in close vicinity to smooth muscle cells and impact its differentiation and development by secreting nerve growth factor (NGF), preadipocyte factor-1 (Pref-1) and insulin-like growth factor-2. It is important to note that NGF is involved in the mechanism of pain and might reflect the severity of the process, which can be useful from the clinical point of view. Preadipocyte factor-1 could be a possible protective factor for further differentiation of cells. Koike et al. concluded that the mast cell might preserve the balance in an affected uterus. ${ }^{28}$ Zhao et al. observed the expression levels of caveolin 1 (CAV1) in adenomyosis-affected tissue and showed that focuses of adenomyosis have a low level of CAV1 expression, which could be associated with adenomyosis-related dysmenorrhea. ${ }^{32}$ The latest data provided by An et al. depicted that macrophages have an impact on epithelial-mesenchymal transition (EMT)-like processes, and thereby could be effector cells in that disease. ${ }^{33}$

Currently, the interest of researchers is focused on abnormal angiogenesis in JZ, which might be involved in the pathogenesis of adenomyosis. Moreover, abnormal vascularization is common for half of the patients: vascular distribution is generally irregular and vessels are thick, dilated, and/or reticular. ${ }^{13}$ Vascular endothelial growth factor (VEGF), fibroblast growth factor (FGF)-1, FGF-2, thrombospondin 1 (TSP-1), and platelet-derived growth factor (PDGF) are potent angiogenic factors. ${ }^{28}$

In general, adenomyosis is characterized by overexpression of VEGF and hypoxia-inducible factor 1-alpha (HIF1-alpha), increasing the number of small vessels. Kang et al. observed 2 alleles of VEGF genes (22578A and 21154A) and concluded that they might be protective factors of adenomyosis. ${ }^{13,34}$ Tokyol et al. emphasized that the quantity and intensity of cyclo-oxygenase (COX-2) expression in the endometrium was growing during both phases of the cycle in patients with adenomyosis in comparison to healthy controls. ${ }^{35}$ Another characteristic feature of the pathology is the prevalence of defective myometrial spiral artery remodeling associated with alteration. ${ }^{36}$

In adenomyosis-induced animal models, the insulin growth factor-II (IGF-II) mRNA was found to be markedly downregulated compared to normal endometrium, as observed by a global gene transcription profiling analysis. Levy et al. proved this with the same tendency of IGF-II expression. ${ }^{21}$ Despite that, its expression in uterine fibroid has the opposite character and this likely indicates different molecular and pathogenic backgrounds of uterine fibroid and adenomyosis. However, the expression of IGF-II can stimulate abnormal myometrial growth in patients with adenomyosis, explaining the frequent coexistence of both pathologies.

\section{Histological features}

Adenomyosis is identified in $20-30 \%$ of all uteri removed at surgery, where it causes globular and cystic enlargement of the myometrium, with some cysts filled with extravasated, often hemolyzed red blood cells and siderophages. ${ }^{5}$ However, in specimens larger than $280 \mathrm{~g}$ it is much less common. ${ }^{4}$ Microscopically, populations of spindle cells include smooth muscle cells and components of adenomyotic lesions, and make contact with the adenomyotic stroma. ${ }^{28}$ On gross examination, the myometrium contains small, soft, red areas, some of which are cystic. Microscopic examination of these lesions reveals glands lined by mildly proliferative to inactive endometrium and surrounded by endometrial stroma. Secretory changes are rare, except during pregnancy and in patients treated with progestins. Myometrial changing (hyperplasia and hypertrophy) could lead to enlarging of the uterus. ${ }^{19}$ Over time, the uterus may also be enlarged from cyclic bleeding into adenomyotic foci. Varying degrees of glandular hyperplasia may be seen, and occasionally hyperplastic surface endometrium extends into the foci of adenomyosis. ${ }^{37}$

Pathological foci are usually seen at a depth of at least $2 \mathrm{~mm}$ in the myometrium on more than 1 microscopic field at $\times 10$ magnification of the JZ. This is useful and important for the uterus during pregnancy and postmenopause. Glands and stroma in adenomyosis are usually in the proliferative phase, whereas they may contain secretory to menstrual transformations. The stromal fibroblasts are 
histologically different from myocytes. ${ }^{5}$ During differential histological analysis, it is important to pay attention to the features of adenomyoma (different from adenomyosis): usually circumscribed, nodular aggregate of smooth muscle, endometrial glands and (usually) endometrial stroma. ${ }^{5}$

\section{Genes and immunity of adenomyosis}

Genetic factors can explain the nature of a variety of diseases, and adenomyosis is no exception. Wang et al. has not revealed any chromosomal aberrations during examination of 25 cases of adenomyosis by comparative genomic hybridization. ${ }^{7}$ Mutations in k-ras and p53 are common in adenomyosis. ${ }^{5}$ Kitawaki has mentioned that Goumenou et al. observed 31 cases of adenomyosis and revealed the incidence of loss of heterozygosity on 2 p22.3p16.1, 3p24.2-p22 and 9p21 chromosomal regions as 19.4\%, 9.7\% and 6.5\%, respectively. ${ }^{7}$ Human leukocyte antigen-DR (HLA-DR) expression is significantly higher in stromal cells compared to glandular cells in adenomyosis tissue. This might be involved in the immune reactions which occur in adenomyosis. ${ }^{38}$

Goumenou et al. observed the expression of p16, retinoblastoma (pRb) and cyclin D1 oncoproteins in patients with adenomyosis. Loss of expression of either pRb or p16, or the overexpression of cyclin D1, could lead to tissue growth. His team showed that for endometrial tissue in patients with adenomyosis during the $1^{\text {st }}$ phase of the cycle, high expression of p16 was and low of pRb is common, whereas for endometrioma this balance was reversed. He concluded that the prevalence of p16 expression was common for adenomyosis in comparison with endometriomas. This clarified the differences in pathogenic mechanisms during both widespread gynecological pathologies. ${ }^{39}$

Fibroblast growth factor 2 (FBG2) is located on chromosome 4q26-27. It can upregulate the expression of VEGF and synergistically act with VEGF in stimulating new vessel formation. Kang et al. demonstrated that the FGF2 754C/G polymorphism is correlated with increased risk of adenomyosis among Chinese women. ${ }^{28,34,40}$

For identification of endometrial stromal cells among the myometrium in adenomyotic foci, immunohistochemical staining for CD10 is used, while it cannot distinguish such pathological conditions as myometrial invasion and carcinoma of endometrium from those involving adenomyosis (in situ). The cells close to the neoplasm also express this marker. ${ }^{5}$

Vimentin expression is lower in adenomyosis in comparison with eutopic endometrium, while expression of $\alpha$-smooth muscle actin and desmin is consistent. ${ }^{28}$

Interleukin-10 (IL-10) is an important immunomodulatory cytokine produced by many cell populations. It is one of the major anti-inflammatory cytokines and plays important role in several chronic inflammatory diseases and cancers. Wang et al. demonstrated that patients with adenomyosis have elevated expression of IL-10 in eutopic and ectopic endometrium (in the secretory phase), which could play a significant role in the pathogenesis of this disease by altering the immune system. ${ }^{41}$ Later, Qin et al. observed the expression of interleukin-10 receptor 1 (IL-10R1) and interleukin-10 receptor 2 (IL-10R2) in adenomyosis. They showed that expression of IL-10R1 and IL-10R2 was higher in adenomyotic samples in comparison with eutopic endometrium of women with adenomyosis or in normal endometrium. They suggested that IL-10 receptors have a possible connection to the immunotolerance and/or antiinflammatory process during adenomyosis. ${ }^{42}$

Leukemia inhibitory factor (LIF) is a pleiotropic cytokine of the interleukin- 6 family, produced by the endometrium during the implantation window. Xiao et al. found significantly lower LIF immunolabeling intensity in women with adenomyosis in comparison to fertile women. The dysregulation of both LIF mRNA and protein in the endometrium during the implantation window suggests that adenomyosis may be associated with impaired implantation. ${ }^{4344}$ Garavaglia et al. noted an overxpression of the human leukocyte antigens (HLA) of class II and an increase of complement components C3 or C4 in adenomyosis. ${ }^{13,31}$

\section{Model systems}

Nowadays, different animal models are widely used for the observation and re-creation of adenomyosis, while for endometriosis only humans and non-human primates are used. ${ }^{45}$ Usually, as the object of experimental models for adenomyosis, non-human primates, horses, dogs, cats, rabbits, and laboratory rodents are used. ${ }^{28}$ This pathology occurs naturally in non-human primates, notably Macaca mulata. Several mouse strains also develop adenomyosis spontaneously and it can be found in a small percentage of adult animals. ${ }^{17,46}$ Moreover, spontaneous development of adenomyosis with a prevalence of $\sim 80 \%$ by 12 months of age was revealed in CD-1 mice. ${ }^{42}$ Adenomyosis has also been induced in mice by hormonal manipulation such as by the implantation of a single anterior pituitary gland into the uterine lumen. ${ }^{17}$ Other experiments were focused on the administration of tamoxifen and further development of adenomyosis in mice. Far fewer studies on adenomyosis have been performed in rats than in mice. Rabbit is a convenient animal model because pathology can spontaneously manifest and its prevalence can be enhanced by estrogen administration during a period of $1-2$ years. ${ }^{45}$

\section{Symptoms}

Adenomyosis does not present pathognomonic clinical symptoms. ${ }^{6,13}$ About $1 / 3$ of women with adenomyosis do not have any symptoms. ${ }^{4,5,12}$ The most frequent symptoms 
are common with other gynecological disorders: menorrhagia, dysmenorrhea, metrorrhagia, dyspareunia, and chronic, erratic or constant pelvic pain. Symptoms of heavy/abnormal bleeding are thought to be positively associated with the depth of penetration of adenomyosis into the myometrium. ${ }^{25,47-49}$ Dysmenorrhea is correlated with the prevalence of adenomyotic glands. ${ }^{6}$

\section{Diagnosis}

In general, symptomatology does not allow detection of adenomyosis in time. The diagnosis can include vaginal examination, transvaginal sonography (TVS) and MRI. ${ }^{18}$ Unfortunately, diagnostic hysteroscopy does not make it possible to discover pathognomonic signs in such cases. ${ }^{12}$ On pelvic examination, the uterus may be enlarged, soft and mildly tender, especially if a patient is examined premenstrually. Ultrasonography is helpful to observe the size and shape of the uterus (a globular or asymmetrical uterus, myometrial cysts), localization of heterogeneous myometrium and of focal abnormal echotexture. Threedimensional reconstruction of the uterine anatomy in the coronal plane provides new and unrivaled views of the JZ. ${ }^{3}$ Sometimes, the adenomyosis may be confined to a part of the myometrium in the form of a well-circumscribed lump, which is called adenomyoma. This is in contrast to the uterine myoma, which does not present a well-defined capsule. ${ }^{15}$

Although pelvic ultrasound is a widely accepted imaging modality, the reported sensitivity for the trans-abdominal approach is only $32-63 \%$ with a corresponding specificity of 95-97\%.$^{50}$ Improvement may be achieved with a transvaginal approach. Hysterosalpingography may be of help, while diverticula extending into the myometrium are present. Magnetic resonance imaging allows observation of the inner myometrium and detection of its thickness

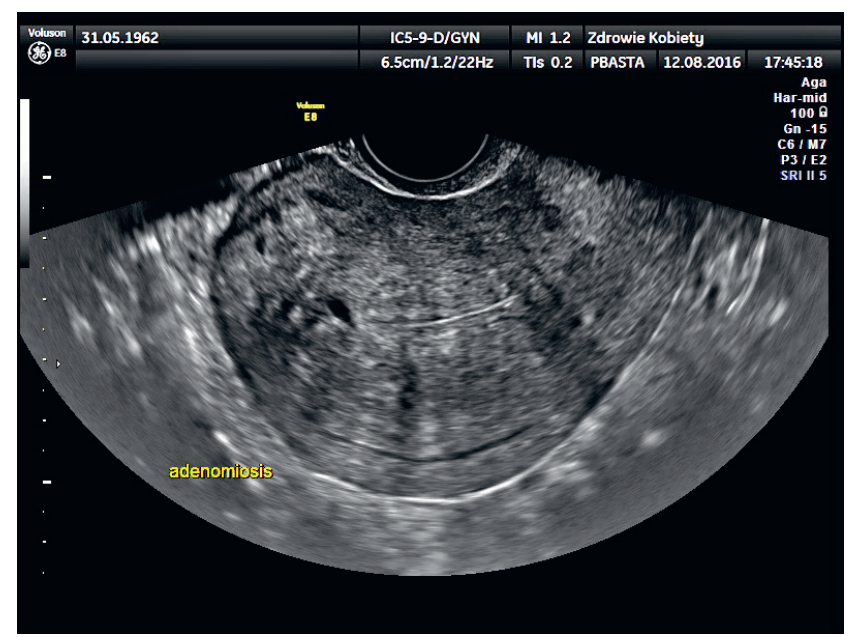

Fig. 1. Transvaginal ultrasound B-mode scan. Sagittal view. Typical changes within the uterus for adenomyosis: enlarged vascular spaces within the inner layer of myometrium and small myometrial cysts (subendometrial cysts - specific sign)

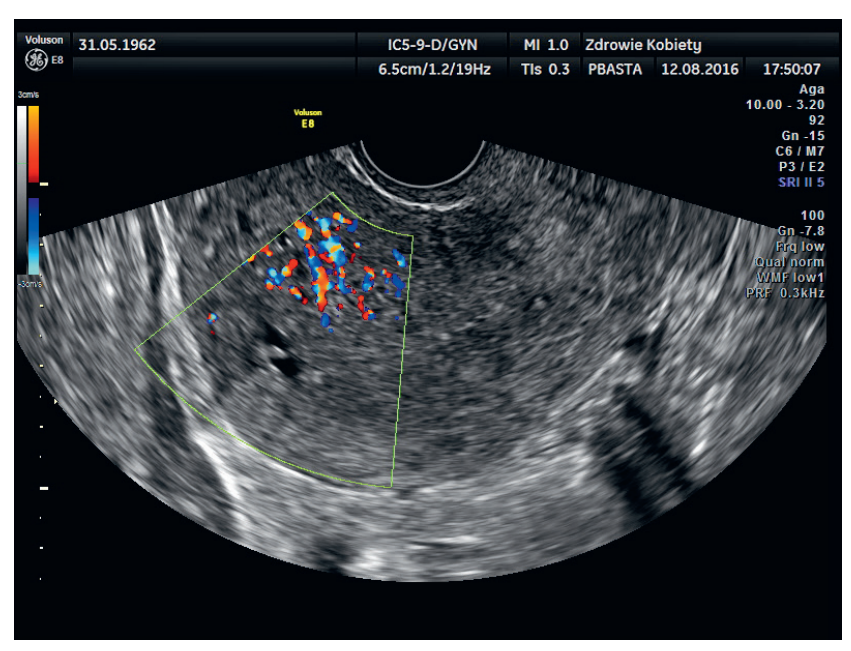

Fig. 2. Transvaginal ultrasound color Doppler scan. Sagittal view. Increased vascularity within myometrium is demonstrated, which is one of the ultrasound adenomyosis features

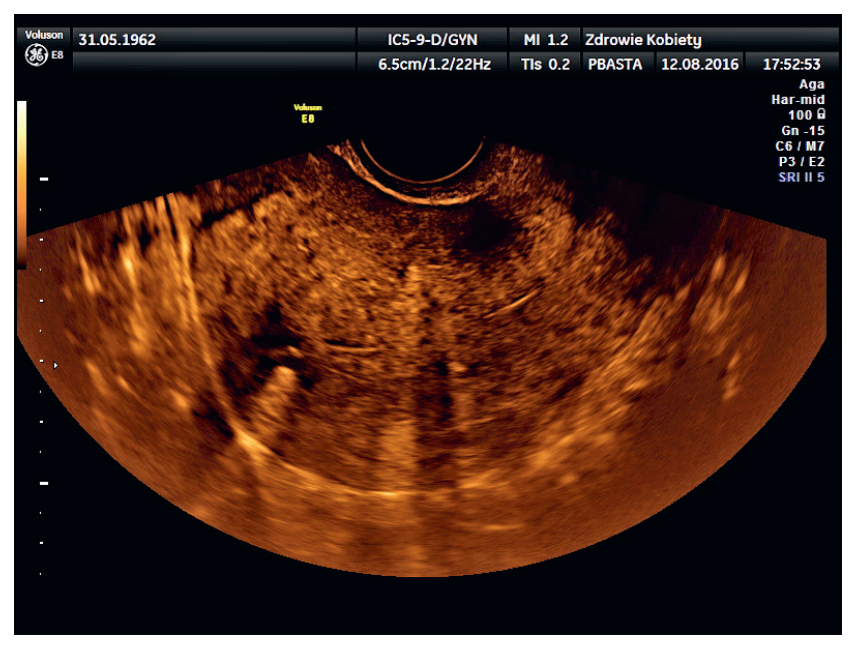

Fig. 3. Transvaginal ultrasound B-mode scan (amber filter). Sagittal view. Typical for adenomyosis changes within the uterus: sub-endometrial echogenic linear striations and acoustic shadowing as a result of ultrasound beam diffraction by irregular structure of myometrium - endometrial tissue causes a hyperplastic reaction ("Venetian blind" appearance)

and nature of changes, which is considered the hallmark of adenomyosis. ${ }^{12}$

Undoubtedly, the histological examination of a few postsurgical uterine transmural sections (from body and fundus) provide the final diagnosis. ${ }^{5}$ Only histological examination can reveal the existence of ectopic glands in the endometrium (the "adeno" component), different from those received by different imaging techniques, which in the majority observe the organization of smooth muscle (the "myosis" component). ${ }^{10}$

\section{Treatment}

The primary indication for treatment is the presence of symptoms that negatively affect woman's daily life. Conservative treatment of pain with nonsteroidal 


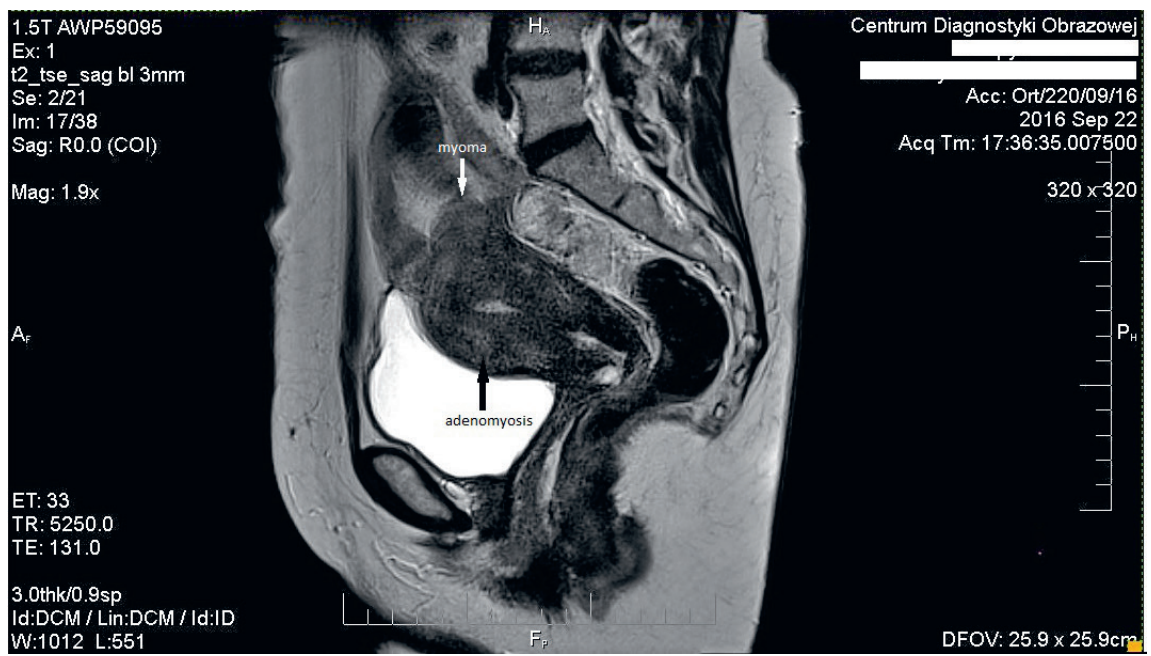

Fig. 4. Contrast-enhanced pelvic magnetic resonance imaging (MRI) of the same patient. T2-weighted image in sagittal section. Magnetic resonance imaging view corresponds with diffuse adenomyosis - high T2 signal regions on the anterior wall of the uterus, representing cystic changes

anti-inflammatory drugs (NSAIDs) and hormonal control of excessive cyclic bleeding maintain the first line of management. Oral progesterone, contraceptive pills, hormonal patches or rings, or levonorgestrel intrauterine device (IUD) can effectively control symptoms. When conservative management is not efficient or contraindicated, surgical manipulations (endometrial ablation/resection, myometrial excision/reduction, myometrial electrocoagulation, uterine artery ligation) are used for the treatment of symptoms. However, these methods are not very effective. ${ }^{9}$ For superficial adenomyosis, using endoscopic endometrial ablation is preferred. ${ }^{5,51}$ The coexistence of uterine fibroids and adenomyosis in 1 patient could complicate the diagnosis as the have a similarity in symptoms. Thus, in both pathologies uterine artery embolization (UAE) is performed. ${ }^{9}$ Less than a half of patients after such surgical treatment have regression in 2-3 years. ${ }^{19}$ Unfortunately, variants with leiomyomata are still complicated to identify among patients with adenomyosis. The former detailed diagnosis of both pathologies might be helpful before UAE, by reason of poor post-surgical prognosis among women with adenomyosis. ${ }^{14}$ If a woman is not a candidate for any medical or surgical conservative management or if that treatment cannot sufficiently control the symptoms, hysterectomy is the final line of treatment.

\section{Outcome}

The junctional zone is quite important for the process of human placentation and is characterized by unique vascular plasticity in terms of physiological remodeling of the myometrial spiral arteries in pregnancy. Increasing thickness of JZ, changes in neo-angiogenesis and alterations in this zone, which are common for adenomyosis and endometriosis, may be associated with a shallow type of defective deep placentation. ${ }^{10-12}$ As it appears from this statement, both pathologies may form the pathogenic background for negative obstetric outcome (preterm birth, fetal growth retardation and postpartum hemorrhage). ${ }^{36}$
Several studies have proved that women with adenomyosis, revealed before pregnancy, had significantly increased risk of preterm delivery, preterm premature rupture of membranes, fetal malpresentation and cesarean delivery. Hypothetically, this could be connected with the enlargement of adenomyotic focuses during pregnancy and further its association with inflammatory consequences. ${ }^{52-54}$ Adenomyosis is connected with impaired implantation, destroying the uterine cavity's receptivity, accompanied by lower expression of the adhesion molecules necessary for embryo implantation (integrin $\beta-3$ and osteopontin). These factors make it possible to evaluate patients with adenomyosis as a target group for in vitro fertilization (IVF) treatment. ${ }^{39,55}$

Neoplastic transformation of adenomyosis is rare: only a few case reports of adenocarcinomas have been published. Koike et al. analyzed all the available data and presented in their review, to date, 44 cases that have been documented. ${ }^{28}$

\section{References}

1. Kido A, Fujimoto K, Matsubara N, Kataoka M, Konishi I, Togashi K. A layer of decreased apparent diffusion coefficient at the endometrial-myometrial junction in uterine adenomyosis. Magn Reson Med Sci. 2015;15(2):220-226.

2. Zhou C, Zhang T, Liu F, et al. The differential expression of mRNAs and long noncoding RNAs between ectopic and eutopic endometria provides new insights into adenomyosis. Mol Biosyst. 2016;12(2): 362-370.

3. Exacoustos C, Brienza L, Di Giovanni A, et al. Adenomyosis: Threedimensional sonographic findings of the junctional zone and correlation with histology. Ultrasound Obstet Gynecol. 2011;37(4):471-479.

4. Levgur M, Abadi MA, Tucker A. Adenomyosis: Symptoms, histology, and pregnancy terminations. Obstet Gynecol. 2000;95(5):688-691.

5. Bergeron C, Amant F, Ferenczy A. Pathology and physiopathology of adenomyosis. Best Pract Res Clin Obstet Gynaecol. 2006;20(4): 511-521.

6. Peric H, Fraser IS. The symptomatology of adenomyosis. Best Pract Res Clin Obstet Gynaecol. 2006;20(4):547-555.

7. Kitawaki J. Adenomyosis: The pathophysiology of an oestrogen-dependent disease. Best Pract Res Clin Obstet Gynaecol. 2006;20(4):493-502.

8. Graziano A, Lo Monte G, Piva I. Diagnostic findings in adenomyosis: A pictorial review on the major concerns. Eur Rev Med Pharmacol Sci. 2015;19(7):1146-1154. 
9. Taran FA, Stewart EA, Brucker S. Adenomyosis: Epidemiology, risk factors, clinical phenotype and surgical and interventional alternatives to hysterectomy. Geburtshilfe Frauenheilkd. 2013;73(9):924-931.

10. Benagiano G, Brosens I. History of adenomyosis. Best Pract Res Clin Obstet Gynaecol. 2006;20(4):449-463.

11. Benagiano G, Brosens I, Lippi D. The history of endometriosis. Gynecol Obstet Invest. 2014;78(1):1-9.

12. Benagiano G, Brosens I, et al. Glob. libr. women's med., (ISSN: 17562228) 2010. doi: 10.3843/GLOWM.10460

13. Di Donato N, Montanari G, Benfenati A, et al. Prevalence of adenomyosis in women undergoing surgery for endometriosis. Eur J Obstet Gynecol Reprod Biol. 2014;181:289-293.

14. Woźniakowska E, Milart P, Paszkowski T. Embolizacja tętnic macicznych - zagadnienia kliniczne. Ginekol Pol. 2013;84:1051-1054.

15. Aleksandrovych V, Bereza T, Sajewicz M, Walocha JA, Gil K. Uterine fibroid: Common features of widespread tumor (Review article). Folia Med Cracov. 2015;55(1):61-75.

16. Verit FF, Yucel O. Endometriosis, leiomyoma and adenomyosis: The risk of gynecologic malignancy. Asian Pac J Cancer Prev. 2013;14(10): 5589-5597.

17. Parrott E, Butterworth M, Green A, White IN, Greaves P. Adenomyosis: A result of disordered stromal differentiation. Am J Pathol. 2001;159(2): 623-630.

18. Naftalin J, Hoo W, Pateman K, Mavrelos D, Holland T, Jurkovic D. How common is adenomyosis? A prospective study of prevalence using transvaginal ultrasound in a gynaecology clinic. Hum Reprod. 2012;27 (12):3432-3439.

19. Romanek K, Bartuzi A, Bogusiewicz M, Rechberger T. Risk factors for adenomyosis in patients with symptomatic uterine leiomyomas. Ginekol Pol. 2010;8:678-680.

20. Shrestha A. Risk factors for adenomyosis. J Nepal Health Res Counc. 2012;10(22):229-233.

21. Levy M, Mittal K, Chiriboga L, Zhang X, Yee H, Wei JJ. Differential expression of selected gene products in uterine leiomyomata and adenomyosis. Fertil Steril. 2007;88(1):220-223.

22. Shen $M$, Liu $X$, Zhang $H$. Transforming growth factor $\beta 1$ signaling coincides with epithelial-mesenchymal transition and fibroblastto-myofibroblast transdifferentiation in the development of adenomyosis in mice. Hum Reprod. 2016;31(2):355-369.

23. Panganamamula UR, Harmanli $\mathrm{OH}$, Isik-Akbay EF, Grotegut CA, Dandolu V, Gaughan JP. Is prior uterine surgery a risk factor for adenomyosis? Obstet Gynecol. 2004;104(5 Pt 1):1034-1038.

24. Curtis KM, Hillis SD, Marchbanks PA, Peterson HB. Disruption of the endometrial-myometrial border during pregnancy as a risk factor for adenomyosis. Am J Obstet Gynecol. 2002;187(3):543-544.

25. Brosens I, Derwig I, Brosens J, Fusi L, Benagiano G, Pijnenborg R. The enigmatic uterine junctional zone: The missing link between reproductive disorders and major obstetrical disorders? Hum Reprod. 2010;25(3):569-574.

26. Hauth EA, Jaeger HJ, Libera $H$, Lange S, Forsting M. MR imaging of the uterus and cervix in healthy women: determination of normal values. Eur Radiol. 2007;17(3):734-742.

27. Tian T, Zhang GF, Zhang H, Liu H. Intravoxel incoherent motion diffusion-weighted imaging in differentiating uterine fibroid from focal adenomyosis: Initial results. Springerplus. 2016;5:9.

28. Koike N, Tsunemi T, Uekuri C, et al. Pathogenesis and malignant transformation of adenomyosis (Review). Oncol Rep. 2013;29(3):861-867.

29. Russell P, Brennan B. Aberrant bcl-2 modulated apoptosis of the endometrium. Pathology. 2005;37:387-389.

30. Zhang L, Li J, Li M. Expression of bcl-2 protein in adenomyosis [in Chinese]. Zhonghua Fu Chan Ke Za Zhi. 2000;35(9):533-535.

31. Garavaglia E, Audrey S, Annalisa I. Adenomyosis and its impact on women fertility. Iran J Reprod Med. 2015;13(6):327-336.

32. Zhao L, Zhou S, Zou L, Zhao X. The expression and functionality of stromal caveolin 1 in human adenomyosis. Hum Reprod. 2013;28(5): 1324-1338.

33. An M, Li D, Yuan M, Li Q, Zhang L, Wang G. Interaction of macrophages and endometrial cells induces epithelial-mesenchymal transition-like processes in adenomyosis. Biol Reprod. 2017;96(1):46-57.
34. Kang S, Li SZ, Wang N, et al. Association between genetic polymorphisms in fibroblast growth factor (FGF)1 and FGF2 and risk of endometriosis and adenomyosis in Chinese women. Hum Reprod. 2010;25(7):1806-1811.

35. Tokyol C, Aktepe F, Dilek FH, Sahin O, Arioz DT. Expression of cyclooxygenase-2 and matrix metalloproteinase-2 in adenomyosis and endometrial polyps and its correlation with angiogenesis. Int J Gynecol Pathol. 2009;28:148-156.

36. Brosens I, Pijnenborg R, Benagiano G. Defective myometrial spiral artery remodelling as a cause of major obstetrical syndromes in endometriosis and adenomyosis. Placenta. 2013;34(2):100-105.

37. Rubin E, Farber JL. Pathology. $2^{\text {nd }}$ ed. Philadelphia, PA: Lippincott; 1988.

38. Koumantakis EE, Panayiotides JG, Goumenou AG, et al. Different HLA-DR expression in endometriotic and adenomyotic lesions: Correlation with transvaginal ultrasonography findings. Arch Gynecol Obstet. 2010;281:851-856.

39. Goumenou AG, Matalliotakis IM, Tzardi M, Fragouli IG, Mahutte NG, Arici A. p16, retinoblastoma (pRb), and cyclin D1 protein expression in human endometriotic and adenomyotic lesions. Fertil Steril. 2006;85(Suppl 1):1204-1207.

40. Kang S, Zhao J, Liu Q, Zhou R, Wang N, Li Y. Vascular endothelial growth factor gene polymorphisms are associated with the risk of developing adenomyosis. Environ Mol Mutagen. 2009;50:361-366.

41. Wang F, Li H, Yang Z, Du X, Cui M, Wen Z. Expression of interleukin-10 in patients with adenomyosis. Fertil Steril. 2009;91(5):1681-1685.

42. Qin X, Zhang H, Wang F, Xue J, Wen Z. Expression and possible role of interleukin-10 receptors in patients with adenomyosis. Eur JObstet Gynecol Reprod Biol. 2010;161(2):194-198.

43. Xiao Y, Sun X, Yang X, et al. Leukemia inhibitory factor is dysregulated in the endometrium and uterine flushing fluid of patients with adenomyosis during implantation window. Fertil Steril. 2010;94(1): 85-89.

44. Tremellen KP, Russell P. The distribution of immune cells and macrophages in the endometrium of women with recurrent reproductive failure. II: Adenomyosis and macrophages. J Reprod Immunol. 2012;93(1):58-63.

45. Greaves P, White IN. Experimental adenomyosis. Best Pract Res Clin Obstet Gynaecol. 2006;20(4):503-510.

46. Chen $Y$, Zhu B, Zhang $H$, et al. Epigallocatechin-3-gallate reduces myometrial infiltration, uterine hyperactivity, and stress levels and alleviates generalized hyperalgesia in mice induced with adenomyosis. Reprod Sci. 2013;20(12):1478-1491.

47. Ates S, Ozcan P, Aydin S. Differences in clinical characteristics for the determination of adenomyosis coexisting with leiomyomas. J Obstet Gynaecol Res. 2016;42(3):307-312.

48. Weiss G, Maseelall P, Schott LL. Adenomyosis a variant, not a disease? Evidence from hysterectomized menopausal women in the Study of Women's Health across the Nation (SWAN). Fertil Steril. 2009;91(1): 201-206.

49. Goluda M, Ujec M, Gabryś M, Kmieciak K. Adenomioza. Wrocław, Poland: Wydawnictwo Cornetis; 2003.

50. Weerakkody Y, Gaillard F, et al. Adenomyosis of the uterus. https:// radiopaedia.org/articles/adenomyosis-of-the-uterus. Accessed on January 12, 2017.

51. Scarperi S, Pontrelli G, Campana C. Laparoscopic radiofrequency thermal ablation for uterine adenomyosis. JSLS. 2015;19(4): pii:e2015.00071. doi: 10.4293/JSLS.2015.00071

52. Kim SC, Lee NK, Yun KY, Joo JK, Suh DS, Kim KH. A rapidly growing adenomyosis associated with preterm delivery and postpartum abscess formation. Taiwan J Obstet Gynecol. 2016;55(4):620-622.

53. Mochimaru A, Aoki S, Oba MS, Kurasawa K, Takahashi T, Hirahara F. Adverse pregnancy outcomes associated with adenomyosis with uterine enlargement. J Obstet Gynaecol Res. 2015;41(4):529-533.

54. Juang CM, Chou P, Yen MS, Twu NF, Horng HC, Hsu WL. Adenomyosis and risk of preterm delivery. BJOG. 2007;114(2):165-169.

55. Harada T, Khine YM, Kaponis A, Nikellis T, Decalvas G, Taniguchi F. The impact of adenomyosis on women's fertility. Obstet Gynecol Surv. 2016;71(9):557-568. 